\title{
MULTI-WAVELENGTH RADIO CONTINUUM EMISSION STUDIES OF DUST-FREE RED GIANTS
}

\author{
Eamon O'Gorman ${ }^{1}$, Graham M. Harper ${ }^{1}$, Alexander Brown ${ }^{2}$, Stepinen Drake ${ }^{3}$, and Anita M. S. Richards ${ }^{4}$ \\ ${ }^{1}$ School of Physics, Trinity College Dublin, Dublin 2, Ireland \\ ${ }^{2}$ Center for Astrophysics and Space Astronomy, University of Colorado, 389 UCB, Boulder, CO 80309, USA \\ ${ }^{3}$ NASA Goddard Space Flight Center, Greenbelt, MD 20771, USA \\ ${ }^{4}$ Jodrell Bank Centre for Astrophysics, School of Physics and Astronomy, University of Manchester, Manchester M13 9PL, UK \\ Received 2013 May 29; accepted 2013 August 5; published 2013 September 12
}

\begin{abstract}
Multi-wavelength centimeter continuum observations of non-dusty, non-pulsating K spectral-type red giants directly sample their chromospheres and wind acceleration zones. Such stars are feeble emitters at these wavelengths, however, and previous observations have provided only a small number of modest signal-to-noise measurements slowly accumulated over three decades. We present multi-wavelength Karl G. Jansky Very Large Array thermal continuum observations of the wind acceleration zones of two dust-free red giants, Arcturus ( $\alpha$ Boo: K2 III) and Aldebaran ( $\alpha$ Tau: K5 III). Importantly, most of our observations of each star were carried out over just a few days, so that we obtained a snapshot of the different stellar atmospheric layers sampled at different wavelengths, independent of any long-term variability. We report the first detections at several wavelengths for each star including a detection at $10 \mathrm{~cm}(3.0 \mathrm{GHz}: S$ band) for both stars and a $20 \mathrm{~cm}(1.5 \mathrm{GHz}: L$ band) detection for $\alpha$ Boo. This is the first time single (non-binary) luminosity class III red giants have been detected at these continuum wavelengths. Our long-wavelength data sample the outer layers of $\alpha$ Boo's atmosphere where its wind velocity is approaching (or possibly has reached) its terminal value and the ionization balance is becoming frozen-in. For $\alpha$ Tau, however, our long-wavelength data are still sampling its inner atmosphere, where the wind is still accelerating probably due to its lower mass-loss rate. We compare our data with published semi-empirical models based on ultraviolet data, and the marked deviations highlight the need for new atmospheric models to be developed. Spectral indices are used to discuss the possible properties of the stellar atmospheres, and we find evidence for a rapidly cooling wind in the case of $\alpha$ Boo. Finally, we develop a simple analytical wind model for $\alpha$ Boo based on our new long-wavelength flux measurements.
\end{abstract}

Key words: radio continuum: stars - stars: chromospheres - stars: individual ( $\alpha$ Boo, $\alpha$ Tau) - stars: late-type stars: winds, outflows

Online-only material: color figures

\section{INTRODUCTION}

In order to understand the mechanisms that drive the $10^{-9}-10^{-11} M_{\odot} \mathrm{yr}^{-1}$ mass-loss rates from evolved spectral-type $\mathrm{K}$ through mid-M stars, an understanding of the dynamics and thermodynamics of their atmospheres is essential. An important discovery in late-type evolved stellar atmospheres resulted from the first ultraviolet survey of such stars using the International Ultraviolet Explorer (IUE). The survey revealed a "transition region dividing line" in the giant branch near spectral type K1 III which separates these stars based on the properties of their atmospheres (Linsky \& Haisch 1979). Stars blueward of the dividing line were found to possess chromospheres and transition regions like the Sun, while stars on the red side were found to possess chromospheres and cool winds. X-ray observations showed that this dividing line extended to coronal emission (Ayres et al. 1981). Around the same time, another class of late-type evolved star emerged which showed signs of possessing both a transition region and a cool wind (e.g., Reimers 1982). Many of these so-called hybrid atmosphere stars now also show evidence for coronal emission, albeit much weaker than on the blue side of the dividing line (Ayres et al. 1997; Dupree et al. 2005). Understanding the nature of the atmospheric structure of late-type evolved stars will ultimately lead to a broader understanding of the mass-loss process.

Mass loss from late-type evolved stars plays a crucial role in both stellar and galactic evolution and ultimately provides part of the material required for the next generation of stars and planets. Despite the importance of this phenomenon and decades of study, the mechanisms that drive winds from evolved spectraltype $\mathrm{K}$ through mid-M stars remain an enduring mystery (clearly laid out by Holzer \& MacGregor 1985 but still unsolved, e.g., Crowley et al. 2009). There is insufficient atomic, molecular, or dust opacity to drive a radiation-driven outflow (Zuckerman et al. 1995; Jones 2008), and acoustic/pulsation models cannot drive the observed mass-loss rates (Sutmann \& Cuntz 1995). Ultraviolet (UV) and optical observations reveal an absence of significant hot wind plasma, and the winds are thus too cool to be Parker-type thermally driven flows (e.g., Linsky \& Haisch 1979; Haisch et al. 1980; Ayres et al. 1981).

Magnetic fields are most likely involved in the mass-loss process, although current magnetic models are also unable to explain spectral diagnostics. Exquisite high signal-to-noise ratio $(\mathrm{S} / \mathrm{N})$ Hubble UV spectra have revealed that the onedimensional (1D) linear Alfvén-wave-driven wind models of the 1980s (e.g., Hartmann \& MacGregor 1980; Harper 1988) are untenable (Harper 2001). These models predict chromospheres as integral parts of a turbulent, extended, and heated wind acceleration zone, but the theoretical line profiles and electron densities do not agree with the Hubble spectra (e.g., Judge \& Carpenter 1998). One important property of cool evolved star winds gleaned from UV spectra is that, for the most part, the red giant winds accelerate in a quasi-steady manner and are not the result of ballistic ejecta as shown by the increase of wind scattering absorption velocity with optical depth in Fe II lines (Carpenter et al. 1999). A new generation of theoretical models 
with outflows driven within diverging magnetic flux tubes has now emerged (Falceta-Gonçalves et al. 2006; Suzuki 2007), but these too are not yet in agreement with observations (Crowley et al. 2009). It has also been suggested that the winds may be driven by some form of magnetic pressure acting on very highly clumped wind material (Eaton 2008), but Harper (2010) does not find compelling evidence for this hypothesis. Progress in this field continues to be driven by observations that provide new insights into the mass-loss problem.

\subsection{Radio Continuum Observations}

Although studies of wind-scattered UV and optical line profiles have provided clues to the mass-loss rates and radial distribution of the mean and turbulent velocity fields, the thermal structure remains poorly constrained. In the UV, the source function, $S_{v}$, of electron collisionally excited emission lines is sensitive to electron temperature, $T_{\mathrm{e}}$ (i.e., $S_{v} \propto e^{-h v / k T_{\mathrm{e}}} / \sqrt{T_{\mathrm{e}}}$ ). Therefore, a localized hot plasma component in a dynamic atmosphere can completely dominate the temporally and spatially averaged emission and hence not reflect the mean radial electron temperature distribution. At radio wavelengths, however, the source function is thermal and is just the Rayleigh-Jeans tail of the Planck function, which is linear in electron temperature (i.e., $S_{v}=2 k T_{\mathrm{e}} v^{2} / c^{2}$ ). This should give a more appropriate estimate of the mean radial electron temperature. It is this value that controls the atomic level populations and ionization of the mean plasma, which is needed to quantify the implied thermal heating supplied to the wind by the unknown driving source/sources, allowing constraints on potential mass-loss mechanisms to be derived.

In the centimeter-radio regime the radio opacity, $\kappa_{\lambda}$, strongly increases with wavelength (i.e., $\kappa_{\lambda} \propto \lambda^{2.1}$ ), and so the longer wavelengths sample the extended layers of a star's atmosphere, thus providing us with spatial information about the star's mass outflow region. The NRAO ${ }^{5}$ Karl G. Jansky Very Large Array (VLA) is sensitive to over three orders of magnitude in continuum optical depth, $\tau_{\lambda}\left(\tau_{20 \mathrm{~cm}} / \tau_{0.7 \mathrm{~cm}} \approx 10^{3}\right)$, and provides an area-averaged sweep through the wind acceleration zone of evolved late-type stars. The thermodynamic properties in this spatial region control the ionization in the far wind because the ionization balance, which also controls the cooling rates, becomes frozen-in at large radii due to advection. Furthermore, it is these outer extended regions of the star's atmosphere that contribute to the commonly seen P Cygni line profiles in the UV. In these profiles the line-of-sight absorption caused by the star's wind is superimposed on the blueshifted scattered emission. Thus, centimeter radio continuum observations can provide a test of models based on these UV profiles. In this paper we directly compare our new VLA observations with atmospheric models derived from UV analysis.

\subsection{Sample Selection}

Currently the most detailed spatial information about the atmospheres of $\mathrm{K}$ and early $\mathrm{M}$ evolved stars is obtained from the $\zeta$ Aurigae and symbiotic eclipsing binaries (e.g., Wright 1970; Baade et al. 1996; Eaton 2008; Crowley et al. 2008). Even though these systems offer us the best opportunity to obtain information on the dynamics and thermodynamics at various heights in the evolved star's atmosphere, the very nature of the

\footnotetext{
5 The National Radio Astronomy Observatory is a facility of the National Science Foundation operated under cooperative agreement by Associated Universities, Inc.
}

Table 1

Stellar and Wind Parameters of $\alpha$ Boo and $\alpha$ Tau

\begin{tabular}{|c|c|c|}
\hline Parameter & $\alpha$ Boo & $\alpha$ Tau \\
\hline Spectral type & K2 III & K5 III \\
\hline HD number & 124897 & 29139 \\
\hline $\operatorname{Mass}\left(M_{\odot}\right)$ & $0.8 \pm 0.2$ & $1.3 \pm 0.3$ \\
\hline Effective temperature (K) & $4294 \pm 30$ & $3970 \pm 49$ \\
\hline Angular diameter (mas) & $21.0 \pm 0.2$ & $20.2 \pm 0.3$ \\
\hline Distance $(\mathrm{pc})$ & $11.3 \pm 0.1$ & $20.4 \pm 0.3$ \\
\hline Radius $\left(R_{\odot}\right)$ & $25.4 \pm 0.3$ & $44.4 \pm 1.0$ \\
\hline Photospheric escape velocity & $110 \mathrm{~km} \mathrm{~s}^{-1}$ & $106 \mathrm{~km} \mathrm{~s}^{-1}$ \\
\hline Rotation period (yr) & $2.0 \pm 0.2$ & 1.8 \\
\hline$[\mathrm{Fe} / \mathrm{H}]$ & $0.5 \pm 0.2$ & $0.15 \pm 0.2$ \\
\hline Wind terminal velocity & $35-40 \mathrm{~km} \mathrm{~s}^{-1}$ & $30 \mathrm{~km} \mathrm{~s}^{-1}$ \\
\hline Mass-loss rate $\left(M_{\odot} \mathrm{yr}^{-1}\right)$ & $2 \times 10^{-10}$ & $1.6 \times 10^{-11}$ \\
\hline Wind temperature $(\mathrm{K})$ & $\sim 10,000$ & $\lesssim 10,000$ \\
\hline Semi-empirical model & Drake (1985) & McMurry (1999) \\
\hline
\end{tabular}

Notes. Masses are from Kallinger et al. (2010) and Lebzelter et al. (2012). Effective temperatures and photospheric angular diameters are from di Benedetto (1993). Distances are from van Leeuwen (2007). Rotaion periods are from Gray \& Brown (2006) and Hatzes \& Cochran (1993). Metallicities are from Decin et al. (2003). Wind parameters are derived from the semi-empirical models of Drake (1985) and Robinson et al. (1998). The Ca II ionization studies of Harper et al. (2004) indicate a wind temperature of $T_{e} \lesssim 1 \times 10^{4} \mathrm{~K}$ for $\alpha$ Tau.

binary system may introduce further complexities. For example, the orbital separation is often within the wind acceleration region, and one could expect flow perturbations to be present (e.g., Chapman 1981). Using the "old" VLA, Harper et al. (2005) find a slow wind acceleration for $\zeta$ Aurigae and confirm that its velocity structure is not typical of single stars with similar spectral types, such as $\lambda$ Velorum (Carpenter et al. 1999).

In order to avoid the assumed additional complexities of a companion, we have selected two single luminosity class III red giants: Arcturus ( $\alpha$ Boo: K2 III) and Aldebaran ( $\alpha$ Tau: K5 III). These nearby red giants have been extensively studied at other wavelengths, and their stellar parameters, which are briefly summarized in Table 1, are accurately known. Both of these late-type giants have "hybrid atmospheres" as they show evidence for both coronal/transition region activity and strong winds. Even though they are slow rotators, three possible values for the mean longitudinal magnetic field (albeit weak: $B=0.65 \pm 0.26,0.43 \pm 0.16$, and $-0.23 \pm 0.20 \mathrm{G})$ have been reported for $\alpha$ Boo (Sennhauser \& Berdyugina 2011) along with a possible magnetic cycle with a period of $\geqslant 14 \mathrm{yr}$ (Brown et al. 2008). Also, the detection of O vi in $\alpha$ Tau (Dupree et al. 2005) indicates magnetic activity in its atmosphere. These stars are predicted to be point sources at all frequencies between 1 and $50 \mathrm{GHz}$ in all VLA configurations, so our radio observations measure their total flux density, $F_{v}$. Moreover, both stars have existing semi-empirical 1D chromospheric and wind models, which we directly compare to our data in this paper.

\section{OBSERVATIONS AND DATA REDUCTION}

Observations of $\alpha$ Boo and $\alpha$ Tau were carried out with the VLA during Open Shared Risk Observing in 2011 February at $Q, \mathrm{Ka}, K, X, C$, and $S$ band in B-configuration (PI: G. M. Harper; Program ID: 10C-105). $\alpha$ Boo was also observed at $S$ and $L$ band in 2012 July when the VLA was again in B-configuration (PI: E. O'Gorman; Program ID: 12A-472). Some details of these observations are given in Table 2. For the 2011 observations, the correlator was set up with two $128 \mathrm{MHz}$ sub-bands centered on the frequencies listed in Table 2. Each sub-band had 64 channels 
Table 2

VLA Observations

\begin{tabular}{|c|c|c|c|c|c|c|c|c|c|}
\hline Star & Date & Band & $\begin{array}{l}\text { Frequency }^{\mathrm{a}} \\
\quad(\mathrm{GHz})\end{array}$ & $\begin{array}{l}\text { Wavelength } \\
(\mathrm{cm})\end{array}$ & $\begin{array}{c}\text { Time on Star } \\
\text { (hr) }\end{array}$ & $\begin{array}{l}\text { Restoring Beam } \\
\quad\left(" \times^{\prime \prime}\right)\end{array}$ & $\begin{array}{c}\text { Bandwidth } \\
(\mathrm{GHz})\end{array}$ & $\begin{array}{l}\text { Number of } \\
\text { Antennae }^{\text {b }}\end{array}$ & $\begin{array}{c}\text { Phase } \\
\text { Calibrator }\end{array}$ \\
\hline \multirow[t]{8}{*}{$\alpha$ Boo } & $2011 \mathrm{Feb} 22$ & $Q$ & 43.3 & 0.7 & 0.3 & $0.19 \times 0.15$ & 0.256 & 22 & $\mathrm{~J} 1357+1919$ \\
\hline & $2011 \mathrm{Feb} 22$ & $\mathrm{Ka}$ & 33.6 & 0.9 & 0.2 & $0.25 \times 0.20$ & 0.256 & 23 & $\mathrm{~J} 1357+1919$ \\
\hline & $2011 \mathrm{Feb} 22$ & $K$ & 22.5 & 1.3 & 0.4 & $0.35 \times 0.28$ & 0.256 & 24 & $\mathrm{~J} 1357+1919$ \\
\hline & $2011 \mathrm{Feb} 11$ & $X$ & 8.5 & 3.5 & 0.3 & $1.14 \times 0.70$ & 0.256 & 18 & $\mathrm{~J} 1415+1320$ \\
\hline & $2011 \mathrm{Feb} 11$ & $C$ & 5.0 & 6.0 & 0.5 & $2.02 \times 1.30$ & 0.256 & 21 & $\mathrm{~J} 1415+1320$ \\
\hline & $2011 \mathrm{Feb} 13$ & $S$ & 3.1 & 9.5 & 1.8 & $2.57 \times 2.08$ & 0.256 & 12 & $\mathrm{~J} 1415+1320$ \\
\hline & 2012 Jul 19 & $S$ & 3.0 & 10.0 & 0.7 & $2.82 \times 2.30$ & 2.0 & 23 & $\mathrm{~J} 1415+1320$ \\
\hline & 2012 Jul 20 & $L$ & 1.5 & 20.0 & 1.6 & $4.46 \times 3.94$ & 1.0 & 23 & $\mathrm{~J} 1415+1320$ \\
\hline \multirow[t]{6}{*}{$\alpha$ Tau } & 2011 Feb 11 & $Q$ & 43.3 & 0.7 & 0.3 & $0.18 \times 0.16$ & 0.256 & 22 & $\mathrm{~J} 0431+1731$ \\
\hline & 2011 Feb 11 & $\mathrm{Ka}$ & 33.6 & 0.9 & 0.2 & $0.22 \times 0.20$ & 0.256 & 19 & $\mathrm{~J} 0449+1121$ \\
\hline & $2011 \mathrm{Feb} 11$ & $K$ & 22.5 & 1.3 & 0.4 & $0.35 \times 0.31$ & 0.256 & 21 & $\mathrm{~J} 0449+1121$ \\
\hline & $2011 \mathrm{Feb} 13$ & $X$ & 8.5 & 3.5 & 0.5 & $0.85 \times 0.78$ & 0.256 & 25 & $\mathrm{~J} 0449+1121$ \\
\hline & $2011 \mathrm{Feb} 13$ & $C$ & 5.0 & 6.0 & 1.2 & $1.48 \times 1.32$ & 0.256 & 21 & $\mathrm{~J} 0449+1121$ \\
\hline & $2011 \mathrm{Feb} 12$ & $S$ & 3.1 & 9.5 & 1.8 & $2.74 \times 2.02$ & 0.256 & 11 & $\mathrm{~J} 0431+2037$ \\
\hline
\end{tabular}

Notes.

${ }^{\text {a }}$ Central frequency of selected bandpass.

b Number of available antennae remaining after flagging.

of width $2 \mathrm{MHz}$ and four polarization products (RR, LL, RL, LR). For the $S$ - and $L$-band observations in 2012, the $1-2 \mathrm{GHz}$ and $2-4 \mathrm{GHz}$ frequency ranges were both divided into 16 subbands, each with 64 channels. The channel width was 2 and $1 \mathrm{MHz}$ for $S$ and $L$ band, respectively.

Both $\alpha$ Boo and $\alpha$ Tau were slightly offset from the phase center by $\sim 5$ synthesized beam widths in order to avoid possible errors at phase center. All scheduling blocks were kept to $\leqslant 2.5 \mathrm{hr}$ of duration. For the high-frequency observations (i.e., $Q$, Ka, and $K$ bands) we used the fast switching technique, which consists of rapidly alternating observations of the target source and a nearby unresolved phase calibrator. The total cycle times for the $Q$-, Ka-, and K-band observations were 160, 230, and 290 s, respectively. For both target sources these high-frequency observations were combined into a single $2 \mathrm{hr}$ observing track and commenced with $X$-band reference pointing with solutions being applied on-line. After $X$-band pointing the target source was observed at $Q$ band to ensure that the best pointing solutions were used. The tracks at lower frequencies were composed of repeatedly interleaved observations of the target source and a nearby phase calibrator but had longer cycle times. The primary calibration sources 3C 286 and 3C 138 were observed at the end of all tracks and were used to measure the complex bandpass and set the absolute flux for $\alpha$ Boo and $\alpha$ Tau, respectively.

The data were flagged, calibrated, and imaged within the Common Astronomical Software Application (CASA; McMullin et al. 2007) package. Data deemed to be bad by the VLA online system were flagged, as were zeros, non-operational antennae, dummy scans at the beginning of each track, and poorly performing antennae. Visual inspection of each scan was carried out to determine if data at the beginning or end of these scans needed to be flagged. For the 2011 low-frequency data the two sub-bands were centered at relatively radio frequency interference (RFI) free regions of the bandpass and only a very small amount of RFI had to be flagged. The 2012 wide-band data were initially Hanning smoothed (combining adjacent frequency channels with weights $0.25,0.5$, and 0.25 ) to suppress Gibbs ringing. We manually flagged entire sub-bands that were badly contaminated with RFI. The testautoflag task was then used to conservatively flag RFI from all sources, and any remaining RFI was manually flagged.
In order to calibrate the data, we solved for the complex gains of the calibration sources while applying the bandpass solution, which was derived from the relevant flux calibrator. The amplitude gains of the phase calibrators were scaled according to values derived from the flux calibrators using the "Perley-Butler 2010" flux density standard (Perley \& Butler 2013). At the time, no Ka- or $S$-band flux density standard models were available, so instead for these we used the $K$ - and $L$-band models, respectively, which were scaled according to their spectral indices. The more frequently observed phase calibrators were then used to calibrate the amplitude and phases of the targets. Atmospheric opacity corrections were also applied to the high-frequency data sets using the average of a seasonal model (based on many years of measurements) and information from the weather station obtained during the observations.

The visibilities were then both Fourier transformed and deconvolved using the CASA clean task in multi-frequency synthesis imaging mode, which separately grids the multiple spectral channels onto the $u-v$ plane and therefore improves the overall $u-v$ coverage. We used natural weighting for maximum sensitivity, and the cell size was chosen so that the synthesized beam was about five pixels across. For the high frequencies it was usually sufficient to place just one CLEAN circle around the target source. For the low frequencies, however, the image sizes were usually set to a few times the size of the primary beam so that nearby strong serendipitous sources could be CLEANed, thus reducing their sidelobe contamination of the final image. These images were CLEANed interactively, taking sky curvature into account, down to about the $3 \sigma$ level with clean boxes placed around sources as they appeared in the residual image. All images were corrected for primary beam attenuation.

In each image the flux density from the unresolved target source was calculated by (1) taking the peak pixel value from the source, (2) manually integrating the flux density around the source, and (3) fitting an elliptical Gaussian model to the source and deriving the integrated flux density using the CASA imfit task. Each of these values along with the image rms noise measured from adjacent background regions and fitting error produced by imfit are given in Table 3 to indicate the quality of 
Table 3

VLA Flux Densities of $\alpha$ Boo and $\alpha$ Tau

\begin{tabular}{|c|c|c|c|c|c|c|c|c|}
\hline Star & Band & $\begin{array}{c}v^{\mathrm{a}} \\
(\mathrm{GHz})\end{array}$ & $\begin{array}{c}\lambda \\
(\mathrm{cm})\end{array}$ & $\begin{array}{c}\text { Peak } F_{v} \\
\left(\mathrm{mJy} \mathrm{beam}^{-1}\right)\end{array}$ & $\begin{array}{c}\text { Integrated } F_{v} \\
(\mathrm{mJy})\end{array}$ & $\begin{array}{c}\text { imfit Integrated } F_{v} \\
(\mathrm{mJy})\end{array}$ & $\begin{array}{c}\text { Image rms } \\
\left(\mathrm{mJy} \mathrm{beam}^{-1}\right)\end{array}$ & $\begin{array}{l}\text { imfit Fitting Error } \\
(\mathrm{mJy})\end{array}$ \\
\hline \multirow[t]{7}{*}{$\alpha$ Boo } & $Q$ & 43.28 & 0.7 & 5.94 & 6.09 & 6.42 & 0.30 & 0.26 \\
\hline & $\mathrm{Ka}$ & 33.56 & 0.9 & 4.16 & 4.32 & 4.49 & 0.08 & 0.09 \\
\hline & $K$ & 22.46 & 1.3 & 1.83 & 1.78 & 1.81 & 0.04 & 0.05 \\
\hline & $X$ & 8.46 & 3.5 & 0.51 & 0.51 & 0.53 & 0.03 & 0.02 \\
\hline & $C$ & 4.90 & 6.1 & 0.21 & 0.14 & 0.16 & 0.04 & 0.01 \\
\hline & $S$ & 2.87 & 10.4 & 0.13 & 0.12 & 0.12 & 0.01 & 0.02 \\
\hline & $L$ & 1.63 & 18.4 & 0.07 & 0.07 & $\ldots$ & 0.01 & $\ldots$ \\
\hline \multirow[t]{5}{*}{$\alpha$ Tau } & $Q$ & 43.28 & 0.7 & 3.67 & 3.73 & 4.08 & 0.26 & 0.18 \\
\hline & $\mathrm{Ka}$ & 33.56 & 0.9 & 2.19 & 1.96 & 2.13 & 0.09 & 0.07 \\
\hline & $K$ & 22.46 & 1.3 & 1.86 & 1.88 & 2.07 & 0.04 & 0.08 \\
\hline & $X$ & 8.46 & 3.5 & 0.30 & 0.29 & 0.28 & 0.01 & 0.02 \\
\hline & $C$ & 4.96 & 6.0 & 0.15 & 0.17 & 0.18 & 0.01 & 0.01 \\
\hline
\end{tabular}

Note. ${ }^{a}$ Frequency of the final image produced using the multi-frequency synthesis imaging mode within CASA's clean task.

each radio map. Both sources are point sources at all frequencies, so the peak flux value given in Table 3 will also be its total flux density value. For weak detections (i.e., $F_{v} \lesssim 5 \sigma$ ) we avoid using the imfit task to obtain a flux density estimate, as this may produce biased parameter estimates (Taylor et al. 1999). The flux density values used in Section 4 are the peak values listed in Table 3. We assume absolute flux density scale systematic uncertainties of 3\% at all frequencies (Perley \& Butler 2013).

\section{RESULTS}

Apart from $\alpha$ Boo at $C$ band and $\alpha$ Tau at $S$ band, detections were made in every sub-band for the 2011 data. For all other bands, the flux densities of the targets in both sub-bands were found to be the same within their uncertainties, so we do not present separate values here. Instead we give the values from the radio maps produced by concatenating the two sub-bands. We present in Table 3 the target flux densities extracted from these concatenated radio maps. In the following two sections we briefly discuss the properties of these radio maps for both targets.

\section{1. $\alpha$ Boo Radio Maps}

High-S/N detections $(>19 \sigma)$ of $\alpha$ Boo were made at 22.5, 33.6, and $43.3 \mathrm{GHz}$. Some residuals of the dirty beam remained in the CLEANed maps due to the paucity of uv-coverage in these short high-frequency observations. At the lower frequencies, it was necessary to image confusing sources, notably a strong radio source located $186^{\prime \prime}$ northwest of $\alpha$ Boo. This non-thermal source was reported by Drake \& Linsky (1986), and their flux density of $25 \mathrm{mJy}$ at $4.9 \mathrm{GHz}$ is in close agreement with our measurement of $23.2 \mathrm{mJy}$ at the same frequency. We find the source to have a spectral index $\alpha\left(F_{v} \propto v^{\alpha}\right)$ of -1.4 between 8.5 and $1.6 \mathrm{GHz}$; its flux density reaches $80.3 \mathrm{mJy}$ at $1.6 \mathrm{GHz}$.

We detected $\alpha$ Boo at $6 \sigma$ in the lower frequency sub-band of $C$ band, at $4.9 \mathrm{GHz}$. The noise was slightly higher and the images were poorer quality in the $C$-band higher frequency subband, with artifacts exceeding $\pm 200 \mu \mathrm{Jy}$, and we cannot report a detection in this sub-band, so values given in Table 3 are taken from the lower frequency sub-band only. We obtain good detections $(>5 \sigma)$ of the star for both epochs at $\sim 3 \mathrm{GHz}$ ( $S$ band), and the peak flux densities agree within their uncertainties. We can therefore safely assume that the $1.5 \mathrm{GHz}$ ( $L$-band) flux density has not changed significantly over that period either and so can safely be included in any analysis. The map at $L$ band was highly contaminated by the sidelobes of the strong source northwest of $\alpha$ Boo, but the star is still detected at the $5 \sigma$ level. There is a slight positional offset of $1^{\prime \prime}$ between the position of the peak flux density at 1.5 and at $3.0 \mathrm{GHz}$ for the 2012 data, which were taken within 1 day of each other. However, the position uncertainties due to noise and phase uncertainties between the directions of the phase reference source and the target are at least $1^{\prime \prime}$, and so we feel that it is highly likely that both detections are of $\alpha$ Boo.

\section{2. $\alpha$ Tau Radio Maps}

The final deconvolved radio maps of $\alpha$ Tau were of excellent quality with the rms noise reaching the predicted noise levels in many cases. The target field at all frequencies was free from strong serendipitous radio sources, and thus the final images were free of the sidelobe contamination that was present in the low-frequency $\alpha$ Boo images. $\alpha$ Tau was the only source in the high-frequency maps, while the brightest source in the low-frequency maps was located 106" north-northeast of $\alpha$ Tau and had flux densities of $0.85,1.35$, and $1.7 \mathrm{mJy}$ at $8.5,5.0$, and $3.5 \mathrm{GHz}$, respectively. Strong detections $(>14 \sigma)$ of $\alpha$ Tau were made at all frequencies between 5.0 and $43.3 \mathrm{GHz}$. Due to the limited number of $S$-band receivers available at the time, a full $2.5 \mathrm{hr}$ track was dedicated to $\alpha$ Tau at $3.1 \mathrm{GHz}$ in order to achieve the required sensitivity to give a possible detection. We report a tentative $3 \sigma$ detection of $\alpha$ Tau at $3.1 \mathrm{GHz}$ when we take its peak pixel value as its total flux density.

\section{DISCUSSION}

\subsection{Results versus Previous Observations}

Prior to and during the early operation of the "old" VLA, a small number of single-dish radio observations reported the detection of flares from single red giants (e.g., Slee et al. 1989). These transient radio events have never been re-observed, however, even with more sensitive interferometers, suggesting that such detections were spurious (e.g., Beasley et al. 1992). The first definitive detection of thermal free-free emission from a luminosity class III single red giant at centimeter wavelengths 
Table 4

Compilation of Previous Radio Observations of $\alpha$ Boo and $\alpha$ Tau $(v \leqslant 250 \mathrm{GHz}$ )

\begin{tabular}{|c|c|c|c|c|c|c|}
\hline Star & $\begin{array}{c}v \\
(\mathrm{GHz})\end{array}$ & $\begin{array}{c}\lambda \\
(\mathrm{cm})\end{array}$ & Date & $\begin{array}{c}F_{v} \\
(\mathrm{mJy})\end{array}$ & $\mathrm{S} / \mathrm{N}$ & Source \\
\hline \multirow[t]{12}{*}{$\alpha$ Boo (K2 III) } & 4.9 & 6.1 & 1983 Jan 21 & 0.39 & 3.0 & Drake \& Linsky (1986) \\
\hline & 4.9 & 6.1 & 1983 May 20 & 0.26 & 3.3 & Drake \& Linsky (1986) \\
\hline & 4.9 & 6.1 & 1983 Dec 26 & $\leqslant 0.18(3 \sigma)$ & $\ldots$ & Drake \& Linsky (1986) \\
\hline & 4.9 & 6.1 & 1984 Mar 17 & 0.24 & 4.8 & Drake \& Linsky (1986) \\
\hline & 15.0 & 2.0 & 1984 Nov 6 & 0.68 & 7.6 & Drake \& Linsky (1986) \\
\hline & 22.5 & 1.3 & 1999 Jan 6 & 1.7 & 8.5 & Dehaes et al. (2011) \\
\hline & 43.3 & 0.69 & 1999 Jan 6 & 3.3 & 8.3 & Dehaes et al. (2011) \\
\hline & 43.3 & 0.69 & 2004 Jan 25 & 3.34 & 41.8 & Dehaes et al. (2011) \\
\hline & 86.0 & 0.35 & 1985 Nov & 21.4 & 3.0 & Altenhoff et al. (1986) \\
\hline & 108.4 & 0.28 & 1997 Nov-2000 Jun & 20.1 & 29.1 & Cohen et al. (2005) \\
\hline & 217.8 & 0.14 & 1997 Nov-2000 Jun & 83.5 & 48.8 & Cohen et al. (2005) \\
\hline & 250.0 & 0.12 & 1986 Dec-1989 Mar & 78.0 & 9.8 & Altenhoff et al. (1994) \\
\hline \multirow[t]{9}{*}{$\alpha$ Tau (K5 III) } & 4.9 & 6.1 & 1983 Jan 21 & $\leqslant 0.27(3 \sigma)$ & $\ldots$ & Drake \& Linsky (1986) \\
\hline & 4.9 & 6.1 & 1984 Nov 6 & $\leqslant 0.22(3 \sigma)$ & $\ldots$ & Drake \& Linsky (1986) \\
\hline & 5.0 & 6.0 & 1997 Sep 27 & $\leqslant 0.07(3 \sigma)$ & $\ldots$ & Wood et al. (2007) \\
\hline & 8.5 & 3.5 & 1997 Sep 27 & 0.28 & 9.3 & Wood et al. (2007) \\
\hline & 14.9 & 2.0 & 1997 Sep 27 & 0.95 & 11.9 & Wood et al. (2007) \\
\hline & 15.0 & 2.0 & 1984 Nov 6 & 0.60 & 6.0 & Drake \& Linsky \\
\hline & 108.4 & 0.28 & 1997 Nov-2000 Dec & 14.0 & 9.6 & Cohen et al. (2005) \\
\hline & 217.8 & 0.14 & 1999 Sep-2000 Dec & 25.8 & 4.6 & Cohen et al. (2005) \\
\hline & 250.0 & 0.12 & 1986 Dec-1987 Jan & 51.0 & 8.5 & Altenhoff et al. (1994) \\
\hline
\end{tabular}

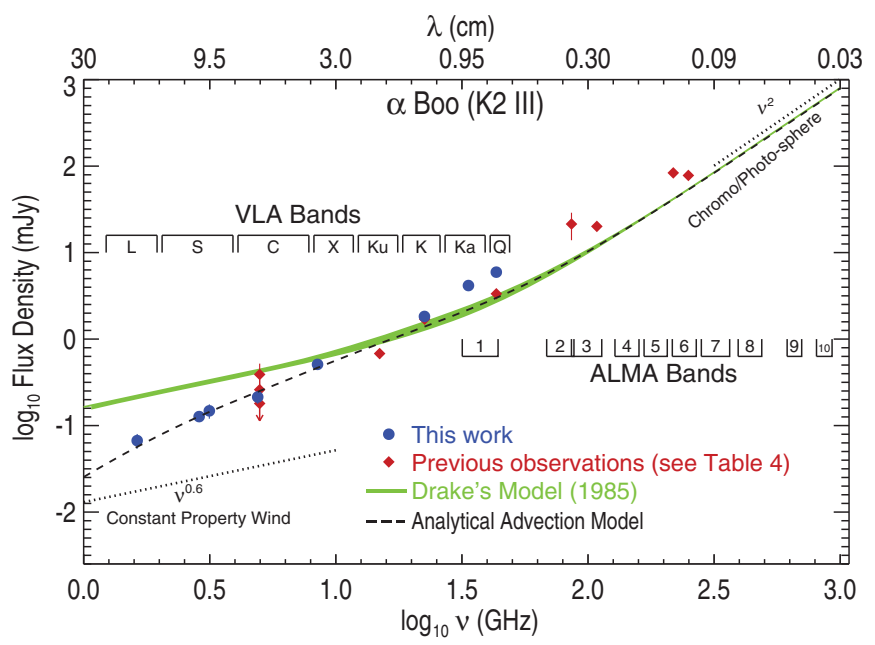

Figure 1. Spectral energy distribution of $\alpha$ Boo for $1 \mathrm{GHz} \leqslant v \leqslant 1 \mathrm{THz}$. Our new multi-frequency VLA observations, which were mainly acquired over a few days in 2011 February, are the blue circles and disagree with the existing chromospheric and wind models of Drake (1985). The overlap between the two models is represented by the green shaded area. The red diamonds are previous observations which were acquired sporadically over the past three decades with the "old" VLA, IRAM, and BIMA. The black dashed line is the expected radio emission from the Drake model which undergoes rapid wind cooling beyond $\sim 2.3 R_{\star}$ (see Sections 4.3 and 4.4).

(A color version of this figure is available in the online journal.)

was of $\alpha$ Boo at $6 \mathrm{~cm}$ (Drake \& Linsky 1983a, 1986). Since then there has been a modest number of centimeter and millimeter observations of this star. In Table 4 we list the majority of these observations and plot their flux densities as a function of frequency in Figure 1. In comparison to other single red giants, $\alpha$ Boo had been relatively well observed at radio continuum wavelengths before this study, including detections in four VLA bands (i.e., $Q, K, \mathrm{Ku}$, and $C$ ). No $\mathrm{Ku}$-band receivers were available during the commissioning phase of the VLA in early 2011, so we can compare three of our detections with previous ones.
Previous detections of $\alpha$ Boo at $6 \mathrm{~cm}$ ranged from a $3 \sigma$ upper limit of $0.18 \mathrm{mJy}$ to a $3 \sigma$ detection at $0.39 \mathrm{mJy}$. Our $6 \mathrm{~cm}$ value agrees to within $\sim 10 \%$ of the highest $\mathrm{S} / \mathrm{N}(5 \sigma)$ value of Drake \& Linsky (1986). There is no significant difference between our $1.3 \mathrm{~cm}$ value and that of Dehaes et al. (2011). There is however a notable difference in flux density values at $0.7 \mathrm{~cm}$, where Dehaes et al. (2011) report values that are lower than ours by over $40 \%$. Although we do not rule out such a level of chromospheric radio variability, it is not expected based on the small level of UV variability observed from such supposedly inactive stars (Harper et al. 2013). Another possibility for the difference in values is that the longer cycle time used by Dehaes et al. (2011), which was over double our value, may lead to larger phase errors and thus lower final flux density values. Future high-frequency VLA observations of $\alpha$ Boo will clarify this discrepancy at $0.7 \mathrm{~cm}$, but past detections at longer wavelengths appear to be in good agreement with our data.

In Figure 2 we plot the previous radio measurements of $\alpha$ Tau at all frequencies below $250 \mathrm{GHz}$ (i.e., $>0.12 \mathrm{~cm}$ ). Prior to this study, $\alpha$ Tau had only been detected at two VLA bands (i.e., $\mathrm{X}$ and $\mathrm{Ku}$ ) and had never been detected at wavelengths longer than $3 \mathrm{~cm}$ due to its relatively low mass-loss rate. Our lack of a Ku-band measurement means that we can only compare the previous $3 \mathrm{~cm}$ detection reported in Wood et al. (2007) to ours. We find that there is no significant difference between the two. Interestingly, Wood et al. (2007) reported a non-detection of $\alpha$ Tau at $6 \mathrm{~cm}$ and placed a $3 \sigma$ upper limit of $0.07 \mathrm{mJy}$ on its emission. In stark contrast to this, we were able to detect the star at $6 \mathrm{~cm}$ with a flux density over two times greater than this value. This hint of variability at long wavelengths would be consistent with the predictions of the broadband nonlinear Alfvén wave model of Airapetian et al. (2010) but can only be confirmed with future high-S/N observations.

\subsection{Existing Atmospheric Models}

One of the most important diagnostic features indicating mass outflows in late-type evolved stars are the blue shifted absorption 


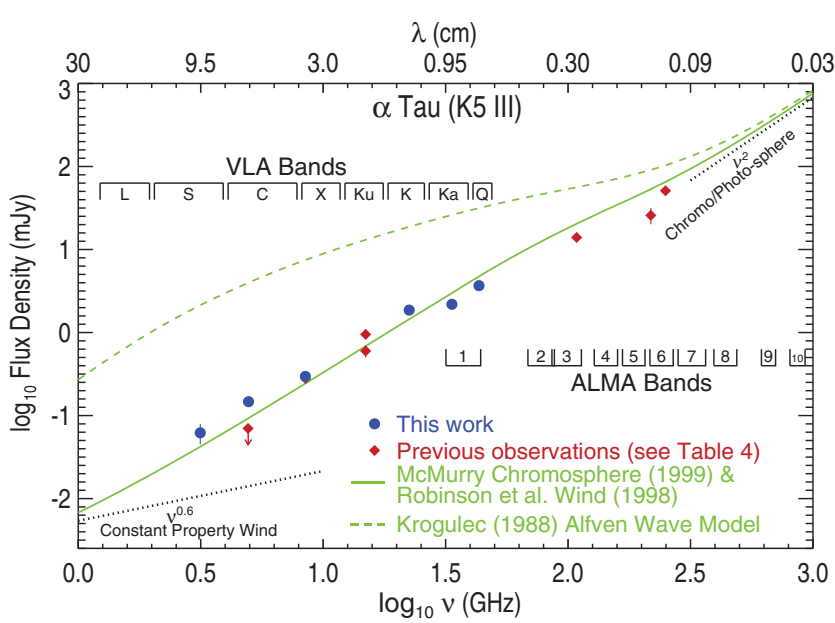

Figure 2. Spectral energy distribution of $\alpha$ Tau for $1 \mathrm{GHz} \leqslant v \leqslant 1 \mathrm{THz}$. Our new multi-frequency VLA observations of $\alpha$ Tau (blue circles) were acquired in just two days in 2011 February. The red diamonds are the previous radio observations of the star which were acquired over many years (see Table 4). The green line is the expected radio emission from the existing hybrid chromosphere and wind model, while the dashed green line is the expected radio emission from a theoretical Alfvén-wave-driven model atmosphere.

(A color version of this figure is available in the online journal.)

components present in the $\mathrm{CaII} \mathrm{H}$ and $\mathrm{K}$ and $\mathrm{Mg} \mathrm{II} \mathrm{h}$ and $\mathrm{k}$ resonance lines. Figure 3 shows one of the two chromosphere and wind models of $\alpha$ Boo (Drake 1985, "model A"), which is based on the Mg II $\mathrm{k} \lambda 2796$ emission line observed with the IUE telescope. The line was modeled by solving the radiative transfer equation in a spherical co-moving frame, and the effects of partial redistribution (e.g., Drake \& Linsky 1983b) were taken into account. Both of Drake's atmospheric models are semi-empirical and contain no assumptions about the winddriving mechanism. They contain the photospheric model of Ayres \& Linsky (1975), predict the wind to reach a terminal velocity of $35-40 \mathrm{~km} \mathrm{~s}^{-1}$ by $2 R_{\star}$, and reach a maximum microturbulence of $5 \mathrm{~km} \mathrm{~s}^{-1}$. They contain a broad temperature plateau with $T_{\mathrm{e}} \approx 8000 \mathrm{~K}$ between 1.2 and $\sim 20 R_{\star}$ with a cooler region farther out, and hydrogen is $\sim 50 \%$ ionized. We compute the radio spectrum from these models assuming spherical 1D geometry (Harper 1994) with the free-free Gaunt factors from Hummer (1988). The radiative transfer equation is solved using the Feautrier technique (Mihalas 1978), and the boundary condition is determined by ensuring that the atmosphere is optically thick at the deepest layers. Drake (1985) predicts that their atmospheric model would produce a flux density value of $0.4 \mathrm{mJy}$ at $6 \mathrm{~cm}$, and encouragingly, our radio spectrum reproduces this value. Departures from spherical symmetry are to be expected in magnetic stellar atmospheres. For example, $\alpha$ Boo has an inclination axis of $58^{\circ} \pm 25^{\circ}$ (Gray \& Brown 2006), and a global magnetic dipole could cause density variations between the equator and the polar regions. Despite this fact, the study of a spherically symmetric atmosphere forms the basis of understanding the more complex environments in real stellar atmospheres.

Figure 1 shows the resulting predicted radio spectrum between $1 \mathrm{GHz}$ and $1 \mathrm{THz}$ for $\alpha$ Boo from these chromosphere and wind models (green line). At high frequencies the radio spectra produced by these models have a blackbody-like slope (i.e., $\sim v^{2}$ ) as a result of the small ion density scale heights close to the star where the temperature is changing slowly. At low frequencies, however, where the Drake models predict the wind to have constant velocity, ionization fraction, and temperature,

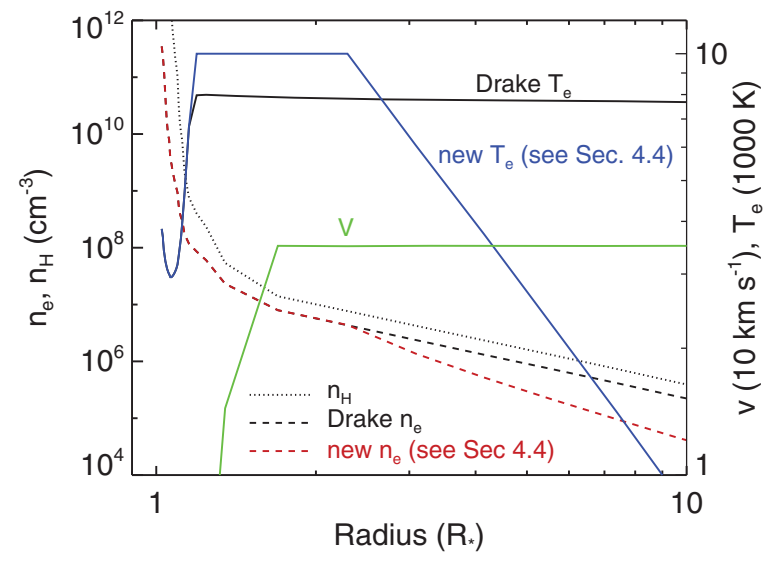

Figure 3. Existing atmospheric model for $\alpha$ Boo (Drake 1985, "model A") along with the same model which undergoes rapid wind cooling beyond $\sim 2.3$ $R_{\star}$ (see Section 4.4). The original Drake models have a temperature plateau of $\sim 8000 \mathrm{~K}$ between 1.2 and $\sim 20 R_{\star}$ (solid black line), reach a terminal velocity of $35-40 \mathrm{~km} \mathrm{~s}^{-1}$ within $2 R_{\star}$ (solid green line), and have a wind which is $50 \%$ ionized (dashed and dotted black lines).

(A color version of this figure is available in the online journal.)

the slope approaches the well-known $\sim \nu^{0.6}$ limit (Wright \& Barlow 1975; Olnon 1975; Panagia \& Felli 1975). The paucity and, in some cases, low $\mathrm{S} / \mathrm{N}$ of previous observations made it difficult to discern the validity of this model prior to our multi-frequency study of $\alpha$ Boo. Our new data reveal significant deviations from the semi-empirical model at both low and high frequencies (in this case below $\sim 8 \mathrm{GHz}$ and above $\sim 25 \mathrm{GHz}$ ). At high frequencies our VLA data indicate a flux excess which is in agreement with previous mm observations. This may be due to larger chromospheric ion densities or to the possible presence of transition region plasma not accounted for in the Drake model. The discrepancy at low frequencies may be due to a lower ionization fraction in the wind, or a lower mass-loss rate than that used in the Drake model.

In Figure 2 we plot the expected radio spectrum of $\alpha$ Tau based on the semi-empirical 1D chromosphere and transition region model of McMurry (1999) embedded in the 1D wind model of Robinson et al. (1998). The semi-empirical McMurry model was created by using the radiative transfer code MULTI (Carlsson 1986) to reproduce the fluxes of collisionally excited C I, C II, Si III, Mg II, and C IV lines in a plane-parallel, hydrostatic, onecomponent atmosphere. It contains the photospheric model of Johnson (1973) and reaches a maximum temperature of $10^{5} \mathrm{~K}$ at $1.2 R_{\star}$. As it does not contain a wind outflow, we use Robinson et al.'s wind characteristics beyond $1.2 R_{\star}$ to describe the outflow velocity. In this wind model, the wind reaches $\sim 80 \%$ of its terminal value of $30 \mathrm{~km} \mathrm{~s}^{-1}$ by $3 R_{\star}$. The Robinson et al. wind characteristics are based on matching the Fe II $\lambda 2755$ line and the O I triplet near $1304 \AA$ with a simplified wind model using the SEI computer code (Lamers et al. 1987). We assume the wind to have a constant temperature of $10,000 \mathrm{~K}$ and have a constant ionization fraction of 0.6 throughout, based on the ionization fraction at the corresponding temperature in the McMurry model.

The radio flux densities at high frequencies (i.e., $v>30 \mathrm{GHz}$ ) are overestimated by the combination of both atmospheric models, although this approach does well in reproducing the VLA flux densities below $30 \mathrm{GHz}$. The VLA, Institut de Radioastronomie Millimétrique (IRAM) $30 \mathrm{~m}$ telescope, and Berkeley Illinois Maryland Association (BIMA) continuum flux 
densities confirm that this model predicts a flux excess at even higher frequencies. One possible explanation for this is that the inner atmosphere contains extensive amounts of cooler gas than that predicted by the 1D static chromosphere and transition region model of McMurry. This scenario agrees with the findings of Wiedemann et al. (1994), who conclude that cool regions exist close to the stellar surface with large ( $>99 \%$ ) filling factors, i.e., a thermally bifurcated CO-mosphere (Ayres \& Rabin 1996). The wind which we have overlain on top of the McMurry chromosphere and transition region is found to be optically thin at nearly all VLA wavelengths, and only contributes a very small flux at the longest wavelengths. As our model matches the data reasonably well below $30 \mathrm{GHz}$, we conclude that $\alpha$ Tau's wind is optically thin and the VLA radio emission at all wavelengths emanates from the inner atmosphere.

We also include the predicted radio spectrum from the theoretical Alfvén-wave-driven outflow model for $\alpha$ Tau (Krogulec 1989) in Figure 2 to demonstrate how radio observations can empirically challenge theoretical models. This model has a fully ionized outflow inside $10 R_{\star}$ and has a mass-loss rate of $6.3 \times$ $10^{-9} M_{\odot} \mathrm{yr}^{-1}$, more than two orders of magnitude higher than the more recent estimate given in Table 1 . As the radio opacity is proportional to $n_{\mathrm{e}} n_{\text {ion }}$, where $n_{\mathrm{e}}$ and $n_{\text {ion }}$ are the electron and ion number densities, respectively, this model greatly overestimates the actual radio flux density at all VLA wavelengths. The linear Alfvén wave models for $\alpha$ Boo (Krogulec 1988) also assume full ionization and have higher mass-loss rates than the value given in Table 1, predicting higher flux densities than observed. The lack of agreement between the Alfvén-wave-driven wind models of Krogulec $(1988,1989)$ and our observed radio fluxes may not necessarily be due to an incorrect wind-driving mechanism and instead may be due to the simplifications and uncertainties in these models, such as wind densities, magnetic field strengths, damping lengths, and flow geometries close to the star. For example, the mass-loss rate is very sensitive to the radial surface magnetic field strength (i.e., $\dot{M} \propto B^{4}$ ) in these Alfvén wave models (Holzer et al. 1983), so a small uncertainty in the mass-loss rate can lead to a large uncertainty in the magnetic field strength. Relaxing some of these simplifications such as purely radial flows or non-assumption of the Wentzel-Kramers-Brillouin approximation (Charbonneau \& MacGregor 1995) may also lead to better agreement with our radio data.

Recently, Ohnaka (2013) has detected a layer of CO in the outer atmosphere of $\alpha$ Tau (i.e., a so-called MOLsphere) which extends out to $\sim 2.5 R_{\star}$, has a temperature of $1500 \pm 200 \mathrm{~K}$, and has a $\mathrm{CO}$ column density of $\sim 1 \times 10^{20} \mathrm{~cm}^{-2}$. They were unable to constrain the geometrical thickness, $\Delta L$, of the MOLsphere from the data, however, and arbitrarily set it to $0.1 R_{\star}$. It can be shown that such a MOLsphere would have an optical depth of $\tau_{6 \mathrm{~cm}}=4.6$ at $C$ band and would produce a corresponding flux density of $0.06 \mathrm{mJy}$, which is considerably lower than our high$\mathrm{S} / \mathrm{N}$ measurement of $0.15 \mathrm{mJy}$. Here, we have conservatively assumed that the electrons in this region of the atmosphere come from singly ionized metals and have an abundance of $\sim 1 \times 10^{-5} n_{\mathrm{H}}$, where $n_{\mathrm{H}}$ is the total hydrogen number density. We have also assumed the CO filling factor to be unity. The disagreement in values between our radio data and the predicted flux for an optically thick disk could mean that the MOLsphere is optically thin at long VLA wavelengths and that the radio emission emanates from the more ionized material closer to the star. It can be shown that the MOLsphere becomes optically thin at $C$ band (i.e., $\tau_{6 \mathrm{~cm}}<1$ ) for $\Delta L>0.46 R_{\star}$, so if the

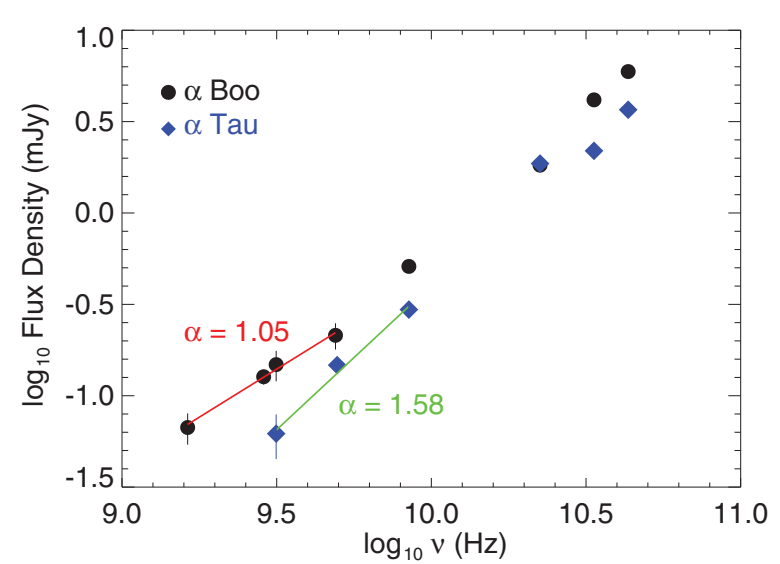

Figure 4. Radio spectra for $\alpha$ Boo and $\alpha$ Tau, together with the best fit power law to their long-wavelength flux densities and the resulting spectral indices. The spectral indices for $\alpha$ Boo and $\alpha$ Tau are found to be 1.05 and 1.58, respectively, which are both larger than the 0.6 value expected for a constant property wind model.

(A color version of this figure is available in the online journal.)

long VLA wavelength radio emission from $\alpha$ Tau comes from a region closer in to the star, then the MOLsphere either has a geometrical width $>0.46 R_{\star}$ or has a filling factor less than unity.

\subsection{Radio Spectral Indices}

Long-wavelength radio emission from non-dusty $\mathrm{K}$ spectraltype red giants is due to thermal free-free emission in their partially ionized outflows, while shorter wavelength radio emission emanates from nearly static lower atmospheric layers. The radio flux density-frequency relationship for these stars is usually found to be intermediate between that expected from the isothermal stellar disk emission, where $\alpha$ follows the RayleighJeans tail of the Planck function (i.e., $\alpha=+2$ ), and that from an optically thin plasma $(\alpha=-0.1)$. It can be shown that the expected radio spectrum from a spherically symmetric isothermal outflow with a constant velocity and ionization fraction varies as $v^{0.6}$ (Wright \& Barlow 1975; Olnon 1975; Panagia \& Felli 1975). If we relax some of the assumptions about the outflow in this constant property wind model and instead assume that the electron density and temperature vary as a function of distance from the star $r$ and have the power-law form $n_{e}(r) \propto r^{-p}$ and $T_{e}(r) \propto r^{-n}$, respectively, then

$$
\alpha=\frac{4 p-6.2-0.6 n}{2 p-1-1.35 n}
$$

(e.g., Seaquist \& Taylor 1987). These power-law approximations are only going to be valid over certain radial ranges of the star's outflow.

The radio spectra for both stars are shown in Figure 4, together with the power laws that were fitted to the long-wavelength flux densities by minimizing the chi-square error statistic. For $\alpha$ Boo a power law with $F_{v} \propto v^{1.05 \pm 0.05}$ fits the four longest wavelength data points well. This spectral index is larger than the 0.8 value obtained by Drake \& Linsky (1986), whose value was based on a shorter wavelength $(2 \mathrm{~cm})$ value and a mean value of four low $\mathrm{S} / \mathrm{N}$ measurements at $6 \mathrm{~cm}$. $\alpha$ Tau was found to have a larger spectral index and a power law with $F_{v} \propto v^{1.58 \pm 0.25}$ best fitted the three longest wavelength data points. This value is in agreement with Drake \& Linsky (1986), who report a value $\geqslant 0.84$, and is lower than the value of 2.18 that can be derived from the shorter wavelength data given in Wood et al. (2007). 


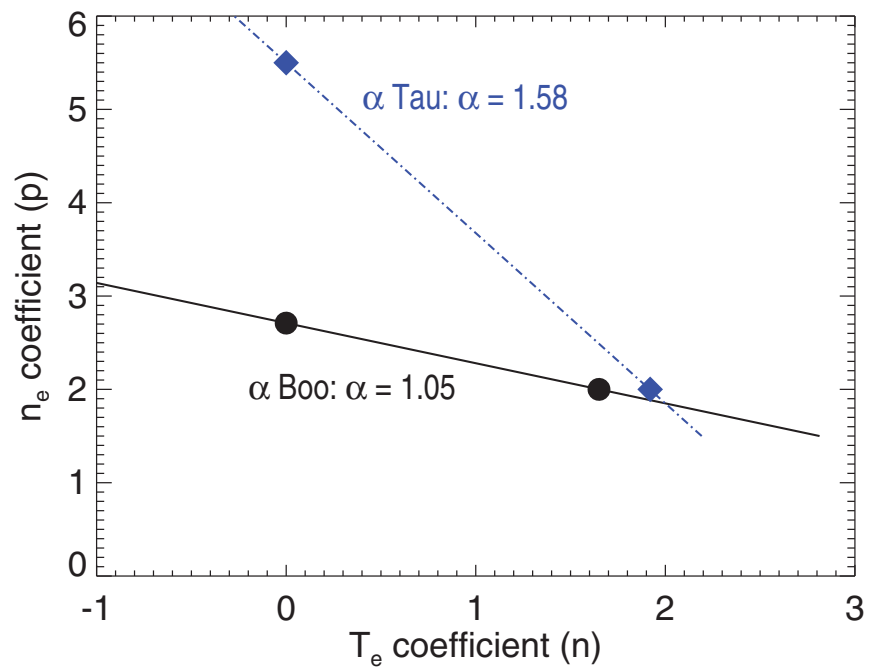

Figure 5. Variation of density and temperature coefficients for the empirically derived spectral indices. The density coefficients for an isothermal flow $(n=0)$ along with the temperature coefficients for a constant outflow velocity $(p=2)$ are also shown for both stars.

(A color version of this figure is available in the online journal.)

It should be emphasized that the spectral index for both stars is steeper than that expected from the constant property wind model.

Equation (1) can be used in conjunction with our new spectral index for each star to calculate the density and temperature coefficients that may describe their outflows. The combinations of the electron temperature and density coefficients are shown for each star in Figure 5 ( $\alpha$ Boo is represented by the solid line and $\alpha$ Tau by the dash-dotted line) along with the coefficients obtained by assuming either an isothermal flow $(n=0)$ or a constant velocity flow $(p=2)$. One potential explanation for spectral indices of stellar outflows being larger than 0.6 is that the wind is still accelerating in the radio emitting region, if the thermal gradients are assumed to be small. For an isothermal flow, the density coefficients are $p=2.7$ and 5.5 for $\alpha$ Boo and $\alpha$ Tau, respectively. From mass conservation, and assuming a steady flow, the power-law coefficients for the velocity profiles of each star can be found, i.e., $v(r) \propto r^{p-2}$. For $\alpha$ Boo we find $v(r) \propto r^{0.7}$, while for $\alpha$ Tau we find $v(r) \propto r^{3.5}$. This suggests that our long VLA wavelengths may probe a steep acceleration region for $\alpha$ Tau's outflow but for $\alpha$ Boo may probe a region where the wind is close to its terminal velocity.

The assumption of shallow thermal gradients in a stellar outflow is probably unreliable, however. It is likely that some form of Alfvèn waves are required to lift the material out of the gravitational potential as suggested by Hartmann \& MacGregor (1980). These waves need damping lengths which are much larger than the chromospheric density scale height $H$ (where $H \sim 0.01 R_{\star}$ for our targets) in order to lift the material out of the gravitational potential, but $\lesssim 1 R_{\star}$ in order to avoid wind terminal velocities greater than those observed (e.g., Holzer et al. 1983). It has also been shown that these waves are expected to produce substantial heating near the base of the wind (e.g., Hartmann et al. 1982). If the longwavelength radio emission from $\alpha$ Tau is indeed emanating from the wind acceleration region, then the dissipation of these Alfvèn waves may introduce thermal gradients in this region and its velocity profile will not be described by $v(r) \propto r^{3.5}$. Furthermore, diverging flow geometries have been invoked as a more realistic representation of stellar atmospheres (Hartmann

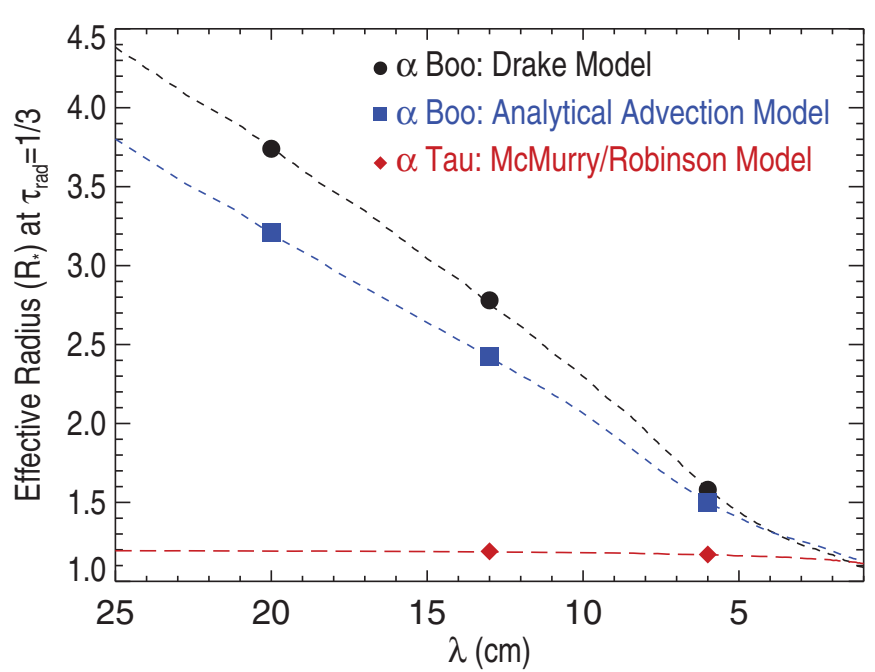

Figure 6. Predicted effective radius (dashed lines) as a function of wavelength derived from the existing atmospheric models of $\alpha$ Boo and $\alpha$ Tau. Also plotted is the predicted effective radius derived from our analytical advection model for $\alpha$ Boo (discussed in Section 4.4). Points corresponding to our long-wavelength VLA measurements are also shown. At the same radio wavelengths the lower mass-loss rate of $\alpha$ Tau results in a smaller effective radius than that for $\alpha$ Boo. (A color version of this figure is available in the online journal.)

\& MacGregor 1982; Jatenco-Pereira \& Opher 1989; Vidotto et al. 2006), and so one could write the area of a flux tube (normalized to its value at $\left.r_{1}\right)$ as $\left(r / r_{1}\right)^{2} f(r)$, where $f(r)$ is a function describing the divergence from a purely radial flow [i.e., when $f(r)=1$ ]. If the non-radial expansion term can be described by a power law $f(r) \propto r^{s}$, where $s>1$ indicates super-radial expansion, then $v(r) \propto r^{3.5-s}$ would describe the velocity profile of $\alpha$ Tau (assuming an isothermal flow with a constant ionization fraction). Therefore, including diverging geometries reduces the magnitude of the acceleration. Farther out in the wind, where it has reached its terminal velocity, one would also expect a thermal gradient (but now of opposite sign) due to adiabatic expansion and line cooling. If the longwavelength radio emission emanates from this region of the wind, then Equation (1) provides us with a direct estimate of the temperature coefficient as we can assume the density coefficient is $p=2$. This may be the case for our long VLA wavelength measurements of $\alpha$ Boo, in which case $T_{e}(r) \propto r^{-1.65}$.

To investigate this matter further, we estimate the effective radius of the radio emitting region as a function of wavelength based on the Drake model for $\alpha$ Boo and the hybrid McMurry and Robinson et al. model for $\alpha$ Tau. We follow the approach used by Cassinelli \& Hartmann (1977) and assume that the radio emission at each wavelength is characterized by emission from a radial optical depth $\tau_{\text {rad }} \sim 1 / 3$. This is a modification of the Eddington-Barbier relation for an extended atmosphere where emission from smaller optical depths has added weight. Since the radio free-free opacity increases at longer wavelengths, the optical depth along a line of sight into the stellar outflow also increases at longer wavelengths. This implies that the effective radius (i.e., the radius where $\tau_{\lambda}=\tau_{\text {rad }}$ ) will increase with longer wavelengths and will be greater for outflows with higher densities of ionized material as $\tau_{\lambda}(r) \propto \lambda^{2.1} \int n_{\text {ion }}(r) n_{\mathrm{e}}(r) d r$.

The larger mass-loss rate of $\alpha$ Boo in comparison to $\alpha$ Tau means that the latter has a substantially smaller effective radius at longer wavelengths, as seen in Figure 6. At 6,13, and $20 \mathrm{~cm}$ the effective radius of $\alpha$ Boo at $\tau_{\text {rad }}=1 / 3$ is predicted to be 1.6 , 2.8 , and $3.7 R_{\star}$ but is only $\sim 1.2 R_{\star}$ at 6 and $13 \mathrm{~cm}$ for $\alpha$ 
Tau. Robinson et al. (1998) predict that $\alpha$ Tau's wind reaches $\sim 80 \%$ of its terminal velocity by $3 R_{\star}$, but even our longest wavelength observations are highly unlikely to sample the wind outside the lower velocity layers closer to the star. For $\alpha$ Boo, however, Drake (1985) predicts that the wind has reached its terminal velocity by $\sim 2 R_{\star}$, so based on this model our longest wavelength measurements are of the region where the wind has reached a steady terminal velocity. From Figure 5, this implies that the $n_{\mathrm{e}}$ coefficient is $p=2$ and thus the $T_{\mathrm{e}}$ coefficient is $n=1.65$. Pure adiabatic spherical expansion cooling with no heat source has $n=1.33$, so additional cooling routes must be operating, possibly due to line cooling. Finally, the wind ionization balance may not have become frozen-in in the region of $\alpha$ Boo's wind where the radio emission emanates from. If this is true, then the excess slope of the spectral index could be due to a combination of both cooling and changing ionization fraction. In this scenario the temperature coefficient $n$ would be smaller than our derived value because Equation (1) assumes a constant ionization fraction.

\subsection{Analytical Advection Model for $\alpha$ Boo's Wind}

A failure of the Drake model for $\alpha$ Boo is that it overestimates the radio fluxes at long VLA wavelengths which sample the outer atmosphere, as clearly shown in Figure 1. If these wavelengths are indeed sampling the wind at its terminal velocity, then one reason for this overestimation is that the wind is cooling closer in than predicted by the existing model, which assumes a constant temperature of $8000 \mathrm{~K}$ out to $\sim 20 R_{\star}$. The main mechanism for such cooling would be adiabatic expansion (O'Gorman \& Harper 2011) and would cause lower electron densities than those predicted by the existing model due to larger recombination rates.

To investigate the possibility of the wind undergoing more rapid cooling closer in to the star, we adjusted one of the existing models (referred to as "Model A" in Drake 1985) to include a temperature power-law falloff of the form

$$
T_{e}(r)=T_{e}\left(r_{1}\right)\left(\frac{r_{1}}{r}\right)^{n},
$$

at some distance $r_{1}$ from the star, and used the temperature coefficient $n=1.65$ obtained from our new VLA data assuming a constant velocity flow (see Figure 5). We introduce the distance $r_{1}$ as the outer limit to ionization processes; at $r>r_{1}$, the ionization fraction is only determined by recombination. To calculate the new electron density in the wind regime where this temperature falloff occurs, we used the analytical expression of Glassgold \& Huggins (1986) to calculate the hydrogen ionization fraction, $x_{\mathrm{H} \text { II }}=n_{\mathrm{H} \text { II }} / n_{\mathrm{H}}$, where $n_{\mathrm{HII}}$ and $n_{\mathrm{H}}$ are the ionized and total hydrogen number densities, respectively. To do so, we need to make a number of assumptions about the wind properties beyond radius $r_{1}$, namely:

1. A constant velocity mass outflow, i.e., $n_{\mathrm{H}}(r)=C / r^{2}$, where $C$ is a constant proportional to the ratio of the mass-loss rate divided by the terminal velocity. For $\alpha$ Boo, $C=1.7 \times 10^{32} \mathrm{~cm}^{-1}$ assuming a wind velocity of $35 \mathrm{~km} \mathrm{~s}^{-1}$.

2. All hydrogen ionization processes cease beyond $r_{1}$. The ionization of hydrogen in the chromosphere and wind is a two-stage process: the $n=2$ level is excited by electron collisions and Ly $\alpha$ scattering, followed by photoionization by the optically thin Balmer continuum. When the temperature begins to decrease in the wind, the collisional excitation rate and thus ionization rate decrease rapidly.
3. Only radiative recombination of hydrogen is considered, and the temperature variation of the recombination coefficient $\alpha_{B}$ which excludes captures to the $n=1$ level (Spitzer 1978) is included. The recombination coefficient varies with temperature as

$$
\alpha_{B}(r)=\alpha_{B}\left(r_{1}\right)\left[\frac{T_{e}\left(r_{1}\right)}{T_{e}(r)}\right]^{0.77}
$$

where the power-law coefficient is obtained by finding the slope of the recombination coefficients between $1000 \mathrm{~K}$ and 16,000 K given in Spitzer (1978).

4. A fixed ion contribution from metals with a low first ionization potential, $x_{\text {ion }}=n_{\text {ion }} / n_{\mathrm{H}}=10^{-4}$, as these are easily ionized in the outflow.

Using these assumptions, it can be shown that the ionization fraction beyond $r_{1}$ is given by (Glassgold \& Huggins 1986)

$$
x_{\mathrm{H} \text { II }}(r)=\frac{x_{\mathrm{HII}}\left(r_{1}\right) x_{\mathrm{ion}} e^{-I(r)}}{x_{\mathrm{ion}}+x_{\mathrm{HII}}\left(r_{1}\right)\left[1-e^{-I(r)}\right]},
$$

where

$$
I(r)=\frac{4.7 \times 10^{9}}{r_{1}}\left[\left(\frac{r_{1}}{r}\right)^{-0.27}-1\right], \text { and } r \geqslant r_{1} .
$$

We adjusted the value of $r_{1}$ to obtain the best fit to our longwavelength observations and found that this happened when $r_{1}=2.3 R_{\star}$. To get this best fit, the existing atmospheric model (plotted in Figure 3) needed to be adjusted, so that it now has a narrower and slightly larger temperature plateau of $T_{e}=10,000 \mathrm{~K}$ between 1.2 and $2.3 R_{\star}$ and a temperature profile and a density profile governed by Equations (2) and (4) beyond $r_{1}=2.3 R_{\star}$, respectively. This gives good agreement with our new long-wavelength VLA data as shown in Figure 1. This new hybrid model, which is plotted along with the original Drake model in Figure 3, still has the original ionization fraction of $x_{\mathrm{H} \text { II }} \approx 0.5$ inside $2.3 R_{\star}$ but now contains an initial rapid decrease in $x_{\mathrm{H} \text { II }}$ beyond $2.3 R_{\star}$, which then freezes-in to a constant value of $\sim 0.04$ beyond $\sim 10 R_{\star}$.

Encouraging as it is that such a simple analytical model can reproduce values close to the observed radio fluxes at long wavelengths, it must be stressed that this hybrid model is just a first-order approximation. It assumes that the excess slope from the radio spectrum is a result of rapid cooling only. It still does not reproduce the radio fluxes at wavelengths shorter than $\sim 3 \mathrm{~cm}$, and therefore a new atmospheric model is still required that can reproduce all of the observed flux densities. To do so, the non-trivial task of simultaneously solving the radiative transfer equation and non-LTE atomic level populations which include advection will be required.

\section{CONCLUSIONS}

We have presented the most comprehensive set of multiwavelength radio continuum observations of two standard luminosity class III red giants to date. This is the first time such stars have been detected at wavelengths longer than $6 \mathrm{~cm}$. Such long-wavelength detections are crucial if one wants to study the outer environments of these partially ionized stellar outflows. Our observations were carried out with the VLA during its commissioning phase when only a fraction of the now available bandwidth was at our disposal. The continuous bandwidth 
coverage between 1 and $50 \mathrm{GHz}$ of the new VLA will allow fast detections of historically weak or undetectable radio continuum luminosity class III red giants at both long and short wavelengths. Previous upper limits will be replaced by firm detections, allowing a greater understanding of their outer atmospheric properties.

The spectral index of both $\alpha$ Tau and $\alpha$ Boo at long wavelengths is found to be greater than that expected from a constant property wind. For $\alpha$ Tau our longest wavelength detections are still sampling emission from an accelerating region within the outflow, while for $\alpha$ Boo the emission probably emanates from a region where the flow is close to, or indeed has reached, its terminal velocity. Using our new VLA data, we have developed a simple analytical model for the outer atmosphere of $\alpha$ Boo which contains a rapid wind cooling profile. Future detailed non-LTE radiative transfer models which include advection are required to match all radio flux densities at all wavelengths.

The data presented in this paper were obtained with the Karl G. Jansky Very Large Array (VLA), which is an instrument of the National Radio Astronomy Observatory (NRAO). The NRAO is a facility of the National Science Foundation operated under cooperative agreement by Associated Universities, Inc. We wish to thank the NRAO helpdesk for their detailed responses to our CASA-related queries. We thank the referee for their careful reading of the manuscript and their valuable comments. This publication has emanated from research conducted with the financial support of Science Foundation Ireland under Grant Number SFI11/RFP.1/AST/3064, and a grant from Trinity College Dublin.

Facility: VLA

\section{REFERENCES}

Airapetian, V., Carpenter, K. G., \& Ofman, L. 2010, ApJ, 723, 1210

Altenhoff, W. J., Huchtmeier, W. K., Schmidt, J., Schraml, J. B., \& Stumpff, P. 1986, A\&A, 164, 227

Altenhoff, W. J., Thum, C., \& Wendker, H. J. 1994, A\&A, 281, 161

Ayres, T. R., Brown, A., Harper, G. M., et al. 1997, ApJ, 491, 876

Ayres, T. R., \& Linsky, J. L. 1975, ApJ, 200, 660

Ayres, T. R., Linsky, J. L., Vaiana, G. S., Golub, L., \& Rosner, R. 1981, ApJ, 250,293

Ayres, T. R., \& Rabin, D. 1996, ApJ, 460, 1042

Baade, R., Kirsch, T., Reimers, D., et al. 1996, ApJ, 466, 979

Beasley, A. J., Stewart, R. T., \& Carter, B. D. 1992, MNRAS, 254, 1

Brown, K. I. T., Gray, D. F., \& Baliunas, S. L. 2008, ApJ, 679, 1531

Carlsson, M. 1986, UppOR, 33

Carpenter, K. G., Robinson, R. D., Harper, G. M., et al. 1999, ApJ, 521, 382

Cassinelli, J. P., \& Hartmann, L. 1977, ApJ, 212, 488

Chapman, R. D. 1981, ApJ, 248, 1043

Charbonneau, P., \& MacGregor, K. B. 1995, ApJ, 454, 901

Cohen, M., Carbon, D. F., Welch, W. J., et al. 2005, AJ, 129, 2836

Crowley, C., Espey, B. R., Harper, G. M., \& Roche, J. 2009, in AIP Conf. Ser. 1094, 15th Cambridge Workshop on Cool Stars, Stellar Systems, and the Sun, ed. E. Stempels (Melville, NY: AIP), 267

Crowley, C., Espey, B. R., \& McCandliss, S. R. 2008, ApJ, 675, 711

Decin, L., Vandenbussche, B., Waelkens, C., et al. 2003, A\&A, 400, 709

Dehaes, S., Bauwens, E., Decin, L., et al. 2011, A\&A, 533, A107

di Benedetto, G. P. 1993, A\&A, 270, 315

Drake, S., \& Linsky, J. 1986, AJ, 91, 602

Drake, S. A. 1985, in Proc. Advanced Research Workshop, Progress in Stellar Spectral Line Formation Theory, Trieste, Italy, 1984 September 4-7 (A8637976 17-90), ed. J. E. Beckman \& L. Crivellari (Dordrecht: Reidel), 351

Drake, S. A., \& Linsky, J. L. 1983a, ApJL, 274, L77
Drake, S. A., \& Linsky, J. L. 1983b, ApJ, 273, 299

Dupree, A. K., Lobel, A., Young, P. R., et al. 2005, ApJ, 622, 629

Eaton, J. A. 2008, AJ, 136, 1964

Falceta-Gonçalves, D., Vidotto, A. A., \& Jatenco-Pereira, V. 2006, MNRAS, 368,1145

Glassgold, A. E., \& Huggins, P. J. 1986, ApJ, 306, 605

Gray, D. F., \& Brown, K. I. T. 2006, PASP, 118, 1112

Haisch, B. M., Linsky, J. L., \& Basri, G. S. 1980, ApJ, 235, 519

Harper, G. M. 1988, PhD thesis, Oxford Univ.

Harper, G. M. 1994, MNRAS, 268, 894

Harper, G. M. 2001, in ASP Conf. Ser. 223, 11th Cambridge Workshop on Cool Stars, Stellar Systems and the Sun, ed. R. J. Garcia Lopez, R. Rebolo, \& M. R. Zapaterio Osorio (San Francisco, CA: ASP), 368

Harper, G. M. 2010, ApJ, 720, 1767

Harper, G. M., Brown, A., Ayres, T., \& Sim, S. A. 2004, in IAU Symp. 219, Stars as Suns: Activity, Evolution and Planets, ed. A. K. Dupree \& A. O. Benz (San Francisco, CA: ASP), 651

Harper, G. M., Brown, A., Bennett, P. D., et al. 2005, AJ, 129, 1018

Harper, G. M., O'Riain, N., \& Ayres, T. R. 2013, MNRAS, 428, 2064

Hartmann, L., Avrett, E., \& Edwards, S. 1982, ApJ, 261, 279

Hartmann, L., \& MacGregor, K. B. 1980, ApJ, 242, 260

Hartmann, L., \& MacGregor, K. B. 1982, ApJ, 257, 264

Hatzes, A. P., \& Cochran, W. D. 1993, ApJ, 413, 339

Holzer, T. E., Fla, T., \& Leer, E. 1983, ApJ, 275, 808

Holzer, T. E., \& MacGregor, K. B. 1985, in Mass Loss from Red Giants, ed. M. Morris \& B. Zuckerman (Astrophysics and Space Science Library, Vol. 117; Dordrecht: Reidel), 229

Hummer, D. G. 1988, ApJ, 327, 477

Jatenco-Pereira, V., \& Opher, R. 1989, A\&A, 209, 327

Johnson, H. R. 1973, ApJ, 180, 81

Jones, M. H. 2008, MNRAS, 387, 845

Judge, P. G., \& Carpenter, K. G. 1998, ApJ, 494, 828

Kallinger, T., Weiss, W. W., Barban, C., et al. 2010, A\&A, 509, A77

Krogulec, M. 1988, AcA, 38, 107

Krogulec, M. 1989, AcA, 39, 51

Lamers, H. J. G. L. M., Cerruti-Sola, M., \& Perinotto, M. 1987, ApJ, 314,726

Lebzelter, T., Heiter, U., Abia, C., et al. 2012, A\&A, 547, A108

Linsky, J. L., \& Haisch, B. M. 1979, ApJL, 229, L27

McMullin, J. P., Waters, B., Schiebel, D., Young, W., \& Golap, K. 2007, in ASP Conf. Ser. 376, Astronomical Data Analysis Software and Systems XVI, ed. R. A. Shaw, F. Hill, \& D. J. Bell (San Francisco, CA: ASP), 127

McMurry, A. D. 1999, MNRAS, 302, 37

Mihalas, D. 1978, Stellar Atmospheres (2nd ed.; San Francisco, CA: Freeman)

O’Gorman, E., \& Harper, G. M. 2011, in ASP Conf. Ser. 448, 16th Cambridge Workshop on Cool Stars, Stellar Systems, and the Sun, ed. C. Johns Krull, M. K. Browning, \& A. A. West (San Francisco, CA: ASP), 691

Ohnaka, K. 2013, A\&A, 553, A3

Olnon, F. M. 1975, A\&A, 39, 217

Panagia, N., \& Felli, M. 1975, A\&A, 39, 1

Perley, R. A., \& Butler, B. J. 2013, ApJS, 204, 19

Reimers, D. 1982, A\&A, 107, 292

Robinson, R. D., Carpenter, K. G., \& Brown, A. 1998, ApJ, 503, 396

Seaquist, E. R., \& Taylor, A. R. 1987, ApJ, 312, 813

Sennhauser, C., \& Berdyugina, S. V. 2011, A\&A, 529, A100

Slee, O. B., Stewart, R. T., Bunton, J. D., et al. 1989, MNRAS, 239,913

Spitzer, L. 1978, Physical Processes in the Interstellar Medium (New York: Wiley), 333

Sutmann, G., \& Cuntz, M. 1995, ApJL, 442, L61

Suzuki, T. K. 2007, ApJ, 659, 1592

Taylor, G. B., Carilli, C. L., \& Perley, R. A., (eds.) 1999, ASP Conf. Ser. 180, Synthesis Imaging in Radio Astronomy II (San Francisco, CA: ASP)

van Leeuwen, F. 2007, A\&A, 474, 653

Vidotto, A. A., Falceta-Gonçalves, D., \& Jatenco-Pereira, V. 2006, SSRv, 122,181

Wiedemann, G., Ayres, T. R., Jennings, D. E., \& Saar, S. H. 1994, ApJ, 423, 806

Wood, B. E., Harper, G. M., Müller, H.-R., Heerikhuisen, J., \& Zank, G. P. 2007, ApJ, 655, 946

Wright, A. E., \& Barlow, M. J. 1975, MNRAS, 170, 41

Wright, K. O. 1970, VA, 12, 147

Zuckerman, B., Kim, S. S., \& Liu, T. 1995, ApJL, 446, L79 